\title{
Epidemiology of Endometriosis in France: A Large, Nation-Wide Study Based on Hospital Discharge Data
}

\author{
Peter von Theobald, ${ }^{1,2}$ Jonathan Cottenet, ${ }^{3}$ Silvia Iacobelli, ${ }^{1,4}$ and Catherine Quantin ${ }^{3,5,6}$ \\ ${ }^{1}$ Centre d'Etudes Périnatales de l'Océan Indien (EA 7388), CHU de la Réunion, Site Sud, 97448 Saint-Pierre, France \\ ${ }^{2}$ Department of Gynecology and Obstetrics, Hospital Felix Guyon, CHU de la Reunion, Site Nord, 9740 Saint Denis, France \\ ${ }^{3} \mathrm{CHRU}$ Dijon, Service de Biostatistique et d'Informatique Médicale (DIM) and Université de Bourgogne Franche-Comté, \\ 21000 Dijon, France \\ ${ }^{4}$ Néonatologie, Réanimation Néonatale et Pédiatrique, CHU de la Réunion, Site Sud, 97448 Saint-Pierre, France \\ ${ }^{5}$ INSERM, CIC 1432 and Dijon University Hospital, Clinical Investigation Center, Clinical Epidemiology/Clinical Trials Unit, \\ 21000 Dijon, France \\ ${ }^{6}$ INSERM UMR 1181 "Biostatistics, Biomathematics, Pharmacoepidemiology and Infectious Diseases" (B2PHI), \\ University Bourgogne Franche-Comté, 21000 Dijon, France \\ Correspondence should be addressed to Peter von Theobald; peter.vontheobald@chu-reunion.fr
}

Received 27 January 2016; Accepted 28 March 2016

Academic Editor: Liselotte Mettler

Copyright (C) 2016 Peter von Theobald et al. This is an open access article distributed under the Creative Commons Attribution License, which permits unrestricted use, distribution, and reproduction in any medium, provided the original work is properly cited.

\begin{abstract}
We aimed to assess the prevalence of hospitalization for endometriosis in the general population in France and in each French region and to describe temporal trends, rehospitalization rates, and prevalence of the different types of endometriosis. The analyses were carried out on French hospital discharge data and covered the period 2008-2012 and a population of 14,239,197 women of childbearing age. In this population, the prevalence of hospitalization for endometriosis was $0.9 \%$, ranging from $0.4 \%$ to $1.6 \%$ between regions. Endometriosis affected $1.5 \%$ of hospitalized women of childbearing age, ranging from $1.0 \%$ to $2.4 \%$ between regions. The number of patients hospitalized for endometriosis significantly increased over the study period $(p<0.01)$. Of these, $4.2 \%$ were rehospitalized at least once at one year: ranging from $2.7 \%$ to $6.3 \%$ between regions. The cumulative rehospitalization rate at 3 years was $6.9 \%$. The types of endometriosis according to the procedures performed were as follows: ovarian (40-50\%), peritoneal $(20-30 \%)$, intestinal $(10-20 \%)$, and ureteral or bladder $(<10 \%)$, with significant differences between regions. This is the first detailed epidemiological study of endometriosis in France. Further studies are needed to assess the reasons for the increasing prevalence of endometriosis and for the significant differences in regional prevalence of this disease.
\end{abstract}

\section{Introduction}

Endometriosis is a frequent illness in young women between 15 and 49 years of age. Its prevalence is estimated at 10 to $50 \%$ in the literature [1-3] but no well-conducted nationwide epidemiological studies are available. Its etiology is unknown. Focal ectopic endometrial cells are located mainly in the pelvis, causing intra-abdominal bleeding, inflammation, adhesions, and retractions due to fibrosis. The main symptoms are pelvic pain (dysmenorrhea, dyspareunia, painful defecation or micturation, and painful ovulation) and infertility (due to fallopian tube lesions, adhesions, or direct toxicity of cytokines released by the ectopic cells). Complications such as bowel occlusion, hydroureteronephrosis, ovarian abscess, rectorrhagia, and hematuria may occur. The symptoms are of variable intensity and not always proportional to the extent of the illness. The disease is frequently asymptomatic [3]. The main types of endometriosis are ovarian endometriosis (endometriomas), peritoneal endometriosis, bowel endometriosis, and ureteral and vesical endometriosis. 
They are frequently found in association depending on the extent of the disease. Less frequently, endometriosis may involve the diaphragm, the pleura, the mediastinum, or the meninges. Diagnosis is difficult and is mainly based on the symptoms, vaginal ultrasound, and MRI imaging, with a delay of 4 to 11 years between the first symptom and diagnosis [4-6]. Diagnosis can only be confirmed by surgery, in most cases by laparoscopy followed by a pathology assessment. Endometriosis is a chronic disease with a recurrence rate of 25 to $50 \%$ after conservative treatment [7-9].

As hospitalization as well as surgical investigation is mandatory for the diagnosis, which frequently initiates treatment and involves the most symptomatic patients, we looked at the French hospital discharge database and the Programme of Medicalisation of Information System (PMSI) to assess the prevalence of hospitalization for endometriosis in the population of France. Our main objective was to assess the prevalence of hospitalization for endometriosis in France and in every region of France. Our other objectives were (i) to assess temporal trends in the number of patients, (ii) to assess rehospitalization rates, and (iii) to describe the prevalence of the different types of endometriosis in every French region.

\section{Materials and Methods}

2.1. Programme of Medicalisation of Information System. PMSI was established in France in 1991 and extended in 1997 to all French healthcare facilities [10]. It has compiled discharge abstracts for every admission since 2008 and is an instrument for the financial management and each hospital's budget, which depends on the medical activity described in the PMSI. Diagnoses identified during the admission are coded according to the 10th edition of the international classification of diseases (ICD-10).

All procedures performed during the hospitalization are coded according to the French Common Classification of Medical Procedures (CCAM). Each hospital produces its own anonymous standardized data, which are then compiled at the national level. PMSI provides a huge amount of epidemiological information concerning hospitalized French patients [11-19].

2.2. Study Design. PMSI abstracts for all patients discharged between 1 January 2008 and 31 December 2012 with a main or associated diagnosis ICD code for endometriosis (N800 to N809) were extracted from the national database. Patients were then separated according to the French region they lived in so as to map the prevalence of endometriosis. Only the first hospitalization was considered and rehospitalizations were analyzed separately. Patients were localized according to their postal code of residence (to assess the prevalence among 1549-year-old females of each region and the prevalence among 15-49-year-old female patients admitted to hospital).

In all patients with a main diagnosis of endometriosis alone (N800 to N809), we identified the rate of procedures performed during hospitalization or in the following year with the codes of the CCAM. We defined "specific procedures" related to the most frequently involved organs like
TABLE 1: List of codes for the "organ specific procedures."

\begin{tabular}{|c|c|c|c|}
\hline Procedures addressing & & CCAM codes & \\
\hline Peritoneal endometriosic & HPNA001 & & \\
\hline Peritoneal endometriosis & HPNC001 & & \\
\hline & JFFA012 & HHFA009 & HJCA001 \\
\hline & JFFA014 & HHFA010 & HJCC001 \\
\hline Bowrel endometriosic & JFFC001 & HHFA011 & HJFA002 \\
\hline Dower enduomitriosis & HHFA002 & HHFA014 & HJFA004 \\
\hline & HHFA006 & HHFA016 & HJFA011 \\
\hline & HHFA008 & HHFA017 & HJFA017 \\
\hline & JCCA003 & & \\
\hline & JCCC003 & & \\
\hline Urotorlondometripci & JCEA001 & & \\
\hline ureteral endometriosis & JCEA002 & & \\
\hline & JCEA003 & & \\
\hline & JCEA005 & & \\
\hline & JDFA011 & & \\
\hline & JDFA017 & & \\
\hline Vesical endometriosis & JDFC023 & & \\
\hline & JDCA003 & & \\
\hline & JDCC016 & & \\
\hline & JJFA002 & JJFC003 & \\
\hline & JJFA003 & JJFC004 & \\
\hline & JJFA004 & JJFC006 & \\
\hline Tubo-ovarian endometriosis & JJFA005 & JJFC008 & \\
\hline & JJFA007 & JJFC009 & \\
\hline & JJFA008 & JJFC010 & \\
\hline & JJFA010 & & \\
\hline
\end{tabular}

the peritoneum, bowel, ureter, bladder, and ovaries and quoted the proportion of each specific procedure among all procedures (Table 1).

2.3. Statistical Analysis. The Chi square test was used to compare the prevalence of hospitalization among regions and to compare the specific procedure rates with the total procedure rates.

To evaluate trends in the number of women with endometriosis from 2008 to 2012, we used a Poisson regression.

2.4. Ethics. This study was approved by the National Committee for Data Protection (registration number 1576793). Written consent was not needed for this study. The data from the PMSI database were transmitted by the national agency for the management of hospitalization data (ATIH number 2015-111111-47-33).

\section{Results}

Hospitalization for a diagnosis (main or associated) of endometriosis occurred in $0.9 \%$ of women of childbearing age (between 15 and 49 years of age) in France during the study period. In fact, in a total population of $14,239,197$ 


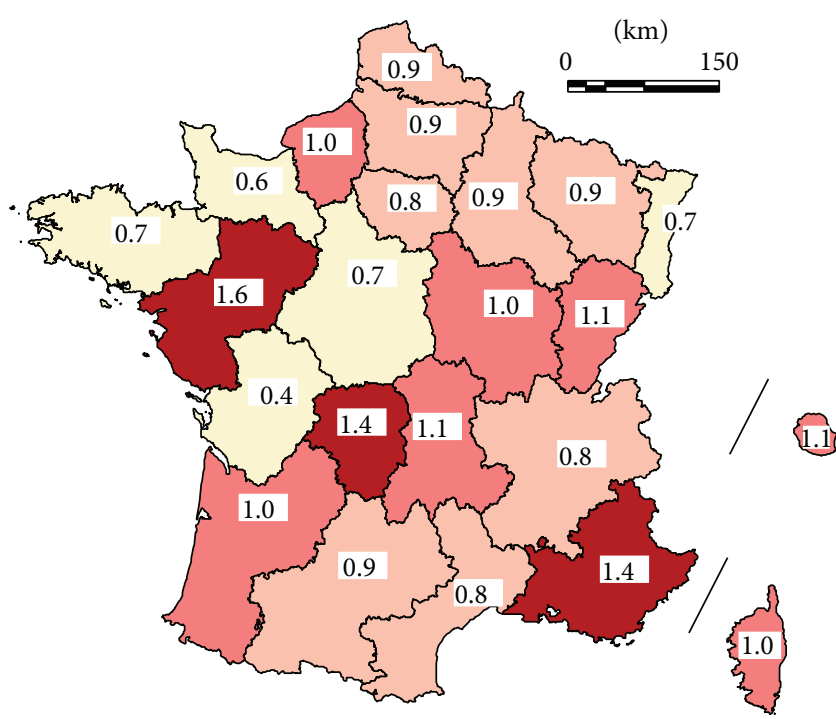

Prevalence of endometriosis among 100 women aged between 15 and 49 years, period 2008-2012
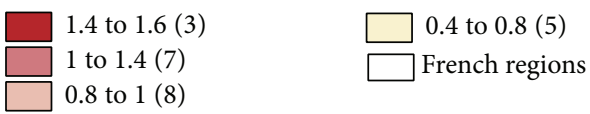

A. Roussot, 2015

Source: national hospital claims database (PMSI) 2008-2012

FIGURE 1: Prevalence of patients hospitalized for main or associated diagnosis of endometriosis in the general population.

women of childbearing age in France, 125,178 patients were hospitalized at least once for endometriosis between 2008 and 2012.

The prevalence of hospitalization for endometriosis in the general population according to region ranged from $0.4 \%$ (Poitou-Charentes) to 1.6\% (Pays de la Loire) (Figure 1). ure 2).

The prevalence in hospital varied in the same way (Fig-

Endometriosis was diagnosed in $1.5 \%$ of hospitalized female patients between 15 and 49 years of age, ranging from $1.0 \%$ (Basse-Normandie) to $2.4 \%$ (Limousin). Comparing Figure 1 (prevalence in the general population) and Figure 2 (prevalence in hospital), evident differences appear. For instance, the regions Pays de la Loire and PACA had the highest prevalence in the general population but a lower prevalence in hospital, and the region Poitou-Charentes had the lowest prevalence in the general population but an aboveaverage prevalence in hospital $(p<0.01)$.

The mean age of the 125,178 patients was $37.9 \pm 8.0$ years.

Concerning trends, the number of patients hospitalized for endometriosis increased significantly $(p<0.01)$ from year to year: from 26,492 in 2008 to 28,322 in 2012 (+6.9\%), while the population of women between 15 and 49 years of age decreased from 14,455,332 in 2008 to 14,239,197 in 2012. Every year, in France, about 458 more patients were hospitalized for endometriosis although the population of women between 15 and 49 years of age fell by an average of 54,034 per year. Of these patients, $4.2 \%$ were rehospitalized

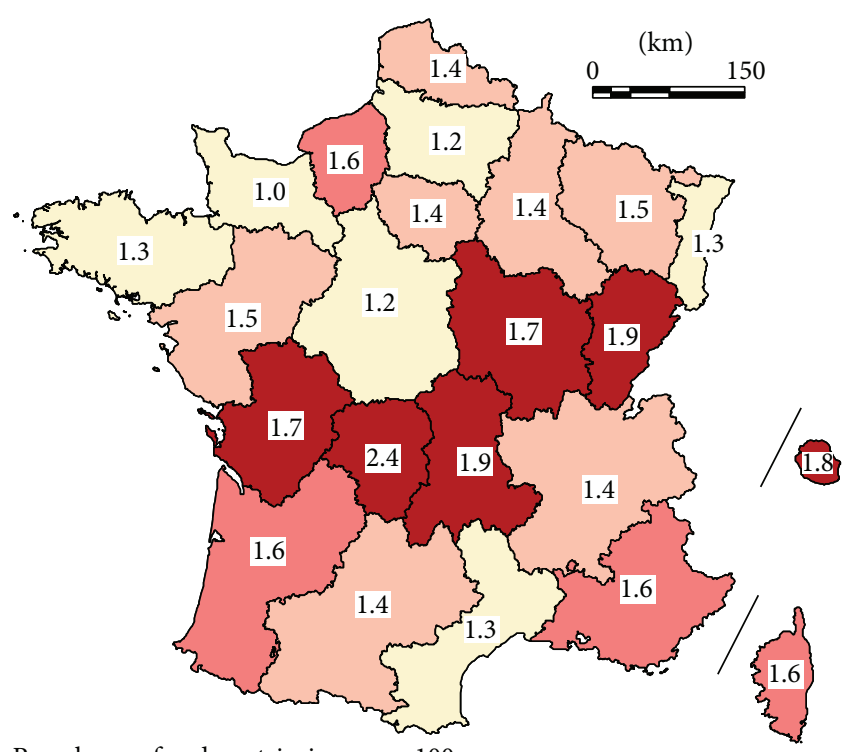

Prevalence of endometriosis among 100 women hospitalized and aged between 15 and 49 years, period 2008-2012

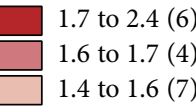

A. Roussot, 2015

Source: national hospital claims database (PMSI) 2008-2012

FIGURE 2: Prevalence of patients hospitalized with a main or associated diagnosis of endometriosis in hospital.

at least once at one year during the study period, ranging from $2.7 \%$ (region Bretagne) to $6.3 \%$ (region Ile-de-France). The cumulative rehospitalization rate in France was 4.1\%, $5.6 \%$, and $6.9 \%$ after 1,2 , and 3 years, respectively, taking into account the fact that all of the patients during the period 2008-2010 had the necessary follow-up of 3 years. The types of endometriosis according to the procedures performed are shown in Figure 3. There were 40 to $50 \%$ of ovarian procedures, mainly removal of endometriomas, 20 to $30 \%$ were procedures for peritoneal lesions, 10 to $20 \%$ concerned extension of the endometriosis to the bowel, and less than $10 \%$ were procedures to cure ureteral or bladder endometriosis. Here again, the rates varied between the regions.

\section{Discussion}

This paper presents the results of the first French study on the epidemiology of endometriosis in a national populationbased setting. Few well-conducted studies have reported data on the prevalence of endometriosis and no data are available on its incidence in women without a previous diagnosis [20-22]. Available data consist of prevalence estimates of diagnosed disease among selected hospital or clinical populations (infertile patients, patients with pelvic pain, etc.) and differences in the reported prevalence of the disease vary by as much as 30-40 times [21-23]. For instance, studies 


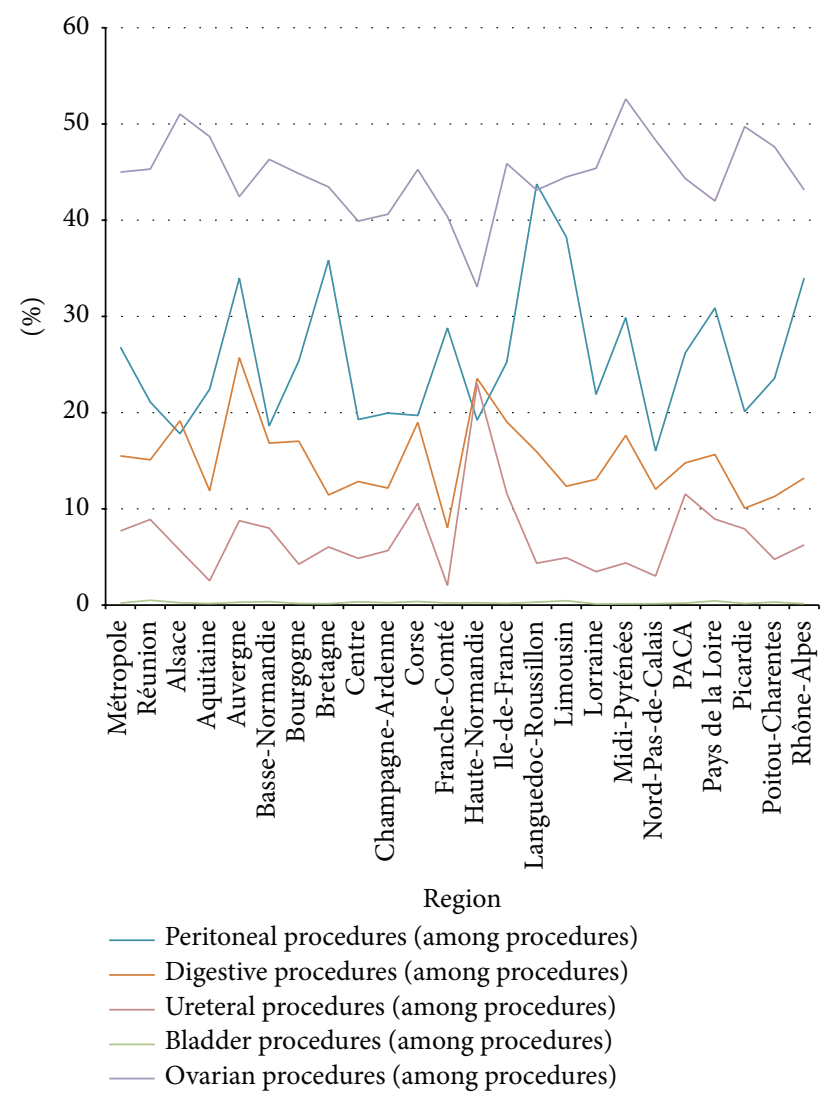

FIgURE 3: Type of endometriosis according to the procedures performed ( $\%$ among procedures).

that analyzed the frequency of endometriosis in women who underwent surgery for fibroids suggested a prevalence of endometriosis of about $10 \%$ [24], but women with fibroids might share the same risk factors as those for endometriosis [25]. Other explanations for these large variations include differences in the indications for surgery, the differing degrees of attention paid by surgeons to the accurate identification of endometriotic lesions, and selective mechanisms that draw patients with suspected endometriosis towards specialized centers $[2,3,20]$.

Formal estimates of the prevalence of pelvic endometriosis in the general female population are lacking [21]. In the literature, there is only one rather old study, somewhat similar to our current work. It is based on diagnoses for patients discharged from short-stay nonfederal hospitals in the United States [26]. This study reported that in 1980 endometriosis was a first-listed diagnosis for 97,000 hospital discharges in females between 15 and 44 years of age.

This figure represented $0.9 \%$ of all first-listed diagnoses, $1.3 \%$ of all first-listed diagnoses minus deliveries, and 6.3\% of all first-listed diagnoses of diseases of the genitourinary system (International Classification of Diseases- (ICD-) 9CM codes 580-629) for females between 15 and 44 years of age. In 1980, women between 15 and 44 years of age in the United States spent an estimated 582,000 days in short-stay nonfederal hospital for health problems for which endometriosis was the first-listed discharge diagnosis. In another study, the annual cost of endometriosis in the United States in 2002 was estimated at $\$ 18.8$ to $\$ 22$ billion [23]. In a recent meta-analysis, the overall direct inpatient costs for patients diagnosed with endometriosis were estimated at $\$ 12,644$ per patient based on the 2002 HCUP database [27]. Our team intends to conduct a further study to estimate the cost of endometriosis in France.

Beyond medicoeconomic studies, it may be interesting to compare the characteristics of regions with very high or very low prevalence in order to identify etiological factors or, at least, risk factors, like the population, pollutants, food habits, or other extrinsic factors. Many of these, mainly pesticide components [28] or other chemicals even in sun lotions [29], have been suspected and assessed in the literature. Of course, genetic factors may also be involved. According to the literature, $30 \%$ of endometriosis patients have a family history of endometriosis [30]. Comparison with other countries using the same discharge data system would be interesting as the prevalence in this study already ranges from $0.4 \%$ to $1.6 \%$ within a single country and as these differences were stable over 5 years.

Our study revealed some marked differences in several regions with regard to prevalence in the general population compared with prevalence in hospital.

These differences were due to other diseases responsible for hospital admissions in the same group of female patients aged 15-49 years, diseases that vary from one region to another. They may also have been caused by the selection of patients: surgery for endometriosis has to be performed in reference centers with a specially trained multidisciplinary team and every region does not have such a surgical department. This means that the two figures give different but complementary information about the prevalence of endometriosis. Our in-hospital prevalence can be compared to the prevalence calculated in the previously cited North American National Center for Health Statistics in 1982 [26]: endometriosis represented $0.9 \%$ of all first-listed diagnoses and in our study, 30 years later on another continent, it was $1.5 \%$. Unfortunately, we do not have the numbers in our population in 1982, but we can suppose that the difference could be explained by the increasing trend found in our series and, possibly, by the improvement in healthcare facilities (imaging techniques, laparoscopy, etc.). It may be surprising that, every year from 2008 to 2012 , about 458 more patients were hospitalized for endometriosis even though the population of women between 15 and 49 years of age fell by 54,034 per year over the same period in France. As hospitalization concerns only the most symptomatic patients (pain, infertility, and complications), it seems likely that the improvement in imaging techniques is not the main explanation for this significant trend, because asymptomatic or paucisymptomatic patients do not undergo surgery. Either tolerance to symptoms like pain or infertility is decreasing in a society where quality of life is improving (and quality of life is severely impaired by endometriosis [5]) or this illness is really becoming more and more frequent. Our study cannot answer this question but it underlines the notion that if there are more patients in hospital, the cost 
of endometriosis will rise. Our study shows the relative frequency of the different types of endometriosis resulting in hospitalization in a nonselected population, or more exactly the entire population of a whole country: the most frequent were ovarian and peritoneal endometriosis. No other reports in the literature based on nationwide data collection describe the proportion of operations related to each pelvic organ involved. It might be interesting to compare our findings with those in other countries to see if the relative proportions are the same or if endometriosis has different patterns. There may be different types of endometriosis, possibly related to different etiologic factors in different geographic areas. The rehospitalization rate increased with time: after 3 years, 6 to $7 \%$ of women had been back in hospital. Our study cannot say whether this was due to a complication, a recurrence, or another reason like in vitro fertilization, for instance. In the literature, recurrence rates vary between 10 and $56 \%$ after 5 years $[31,32]$ and our rehospitalization rate may appear very low compared with published recurrence rates. However, many recurrent patients do not undergo repeat surgery but are treated medically at home.

There may be some limitations to our study. Given the reliance on ICD-10 codes for the selection of patients and the ascertainment of outcomes, there was a potential for underdetection-related biases for endometriosis even though this disease, when diagnosed, is considered debilitating by gynecologists and thus coded. Coding practices may vary among institutions. Nevertheless, coding quality is checked by medical information professionals in each hospital to correct diagnoses and to increase the recorded comorbidity level. Moreover, as many patients are not admitted to hospital, this study did not assess the prevalence of endometriosis in the whole population but only the prevalence of hospitalization for endometriosis. In fact, the diagnosis of endometriosis is very difficult and frequently delayed [4$6,23]$. This may explain the age of the patients at the time of their hospitalization (almost 38 years). Studies have shown the existence of asymptomatic forms of endometriosis [3, 3335]. On the other hand, many endometriotic patients suffer from comorbidities, such as adenomyosis, irritable bowel syndrome, and interstitial cystitis, which can all contribute to the symptomatology. The diagnosis of endometriosis can only be confirmed at surgery (usually laparoscopy) as there are no noninvasive diagnostic methods to effectively screen for endometriosis. Pelvic ultrasound and MRI may lead to a suspicion of the disease and sometimes quantify its invasiveness, but these are limited to moderate or severe forms of the disease and are not suitable for the detection of minimal or mild endometriosis. No biomarker has been identified to date [23]. This means that assessing the prevalence of endometriosis in the general population is very difficult because of the complexity of the diagnosis; it may thus be underestimated. The prevalence obtained from the discharge data is interesting because it concerns only the most symptomatic patients, who require hospitalization and thus have surgical proof of endometriosis. These data are robust and are not based on selected categories of patients; they reveal trends in disease prevalence and make it possible to calculate hospital inpatient costs. Another limitation concerns the difficulty of analyzing all of the factors that may explain differences between the French regions. Further research may be needed, including local investigations to collect information that is not available in our data.

\section{Conclusion}

This nationwide study is the first French study to estimate the prevalence of hospitalization for a main or associated diagnosis of endometriosis in each region of the country $(0.4 \%$ to $1.6 \%)$. It revealed a significant trend towards an increase in hospitalizations for endometriosis in France with time. This study also provides information about the relative proportion of procedures for the different types of endometriosis and addresses the question of rehospitalization. This work may be considered the first of many more-detailed epidemiological studies of endometriosis in France in order to study risk factors and to assess the cost of endometriosis in France.

\section{Competing Interests}

The authors declare that they have no competing interests.

\section{References}

[1] A. E. Schindler, "Pathophysiology, diagnosis and treatment of endometriosis," Minerva Ginecologica, vol. 56, no. 5, pp. 419435, 2004.

[2] P. G. Wardle and M. G. Hull, "1 Is endometriosis a disease?" Bailliere's Clinical Obstetrics and Gynaecology, vol. 7, no. 4, pp. 673-685, 1993.

[3] J. M. R. Rawson, "Prevalence of endometriosis in asymptomatic women," The Journal of Reproductive Medicine, vol. 36, no. 7, pp. 513-515, 1991.

[4] S. Hanssens, C. Rubod, O. Kerdraon et al., "Pelvic endometriosis in women under 25: a specific management?" Minerva Medica, vol. 106, no. 3, pp. 123-131, 2015.

[5] M. Moradi, M. Parker, A. Sneddon, V. Lopez, and D. Ellwood, "Impact of endometriosis on women's lives: a qualitative study," BMC Women's Health, vol. 14, article 123, 2014.

[6] R. O. Burney, "Biomarker development in endometriosis," Scandinavian Journal of Clinical and Laboratory Investigation, vol. 74, supplement 244, pp. 75-81, 2014.

[7] B. Fagervold, M. Jenssen, L. Hummelshoj, and M. H. Moen, "Life after a diagnosis with endometriosis-a 15 years follow-up study," Acta Obstetricia et Gynecologica Scandinavica, vol. 88, no. 8, pp. 914-919, 2009.

[8] M. Moscarini, G. N. Milazzo, C. Assorgi, A. Pacchiarotti, and D. Caserta, "Ovarian stripping versus cystectomy: recurrence of endometriosis and pregnancy rate," Archives of Gynecology and Obstetrics, vol. 290, no. 1, pp. 163-167, 2014.

[9] M.-L. Kim, J. M. Kim, S. J. Seong, S. Y. Lee, M. Han, and Y. J. Cho, "Recurrence of ovarian endometrioma after second-line, conservative, laparoscopic cyst enucleation," American Journal of Obstetrics and Gynecology, vol. 210, no. 3, pp. 216.el-216.e6, 2014.

[10] Ministère de la Santé, "Décret n94-666 du 27 juillet 1994 relatif aux systèmes d'information médicale et à l'analyse de l'activité des établissements de santé publics et privés," Journal Officiel de la République Française, no. 180, Article ID 11395, 1994. 
[11] C. Quantin, E. Benzenine, M. Hägi et al., "Estimation of national colorectal-cancer incidence using claims databases," Journal of Cancer Epidemiology, vol. 2012, Article ID 298369, 7 pages, 2012.

[12] C. Quantin, J. Cottenet, A. Vuagnat et al., "Quality of perinatal statistics from hospital discharge data: comparison with civil registration and the 2010 National Perinatal Survey," Journal de Gynecologie Obstetrique et Biologie de la Reproduction, vol. 43, no. 9, pp. 680-690, 2014.

[13] L. Lorgis, J. Cottenet, G. Molins et al., "Outcomes after acute myocardial infarction in HIV-infected patients: analysis of data from a French nationwide hospital medical information database," Circulation, vol. 127, no. 17, pp. 1767-1774, 2013.

[14] M. Hanf, C. Quantin, P. Farrington et al., "Validation of the French national health insurance information system as a tool in vaccine safety assessment: application to febrile convulsions after pediatric measles/mumps/rubella immunization," Vaccine, vol. 31, no. 49, pp. 5856-5862, 2013.

[15] M. Gusmano, V. Rodwin, D. Weisz, J. Cottenet, and C. Quantin, "Comparison of rehospitalization rates in France and the United States," Journal of Health Services Research \& Policy, vol. 20, pp. 18-25, 2015.

[16] C. Quantin, E. Benzenine, M. Velten, F. Huet, C. Paddy Farrington, and P. Tubert-Bitter, "Self-controlled case series and misclassification bias induced by case selection from administrative hospital databases: application to febrile convulsions in pediatric vaccine pharmacoepidemiology," American Journal of Epidemiology, vol. 178, no. 12, pp. 1731-1739, 2013.

[17] C. Lainay, E. Benzenine, J. Durier et al., "Hospitalization within the first year after stroke: the dijon stroke registry," Stroke, vol. 46, no. 1, pp. 190-196, 2015.

[18] C. Abdulmalak, J. Cottenet, G. Beltramo et al., "Haemoptysis in adults: a 5-year study using the French nationwide hospital administrative database," European Respiratory Journal, vol. 46, no. 2, pp. 503-511, 2015.

[19] A. A. Chantry, C. Deneux-Tharaux, C. Cans, A. Ego, C. Quantin, and M.-H. Bouvier-Colle, "Hospital discharge data can be used for monitoring procedures and intensive care related to severe maternal morbidity," Journal of Clinical Epidemiology, vol. 64, no. 9, pp. 1014-1022, 2011.

[20] P. Viganò, F. Parazzini, E. Somigliana, and P. Vercellini, "Endometriosis: epidemiology and aetiological factors," Best Practice \& Research: Clinical Obstetrics and Gynaecology, vol. 18, no. 2, pp. 177-200, 2004.

[21] D. E. Houston, "Evidence for the risk of pelvic endometriosis by age, race and socioeconomic status," Epidemiologic Reviews, vol. 6, pp. 167-191, 1984.

[22] B. Eskenazi and M. L. Warner, "Epidemiology of endometriosis," Obstetrics and Gynecology Clinics of North America, vol. 24, no. 2, pp. 235-258, 1997.

[23] P. A. W. Rogers, T. M. D’Hooghe, A. Fazleabas et al., "Priorities for endometriosis research: recommendations from an international consensus workshop," Reproductive Sciences, vol. 16, no. 4, pp. 335-346, 2009.

[24] Gruppo Italiano per lo Studio dell'Endometriosi, "Prevalence and anatomical distribution of endometriosis in women with selected gynaecological conditions: results from a multicentric Italian study," Human Reproduction, vol. 9, pp. 1158-1162, 1994.

[25] F. Parazzini, C. La Vecchia, E. Negri, G. Cecchetti, and L. Fedele, "Epidemiologic characteristics of women with uterine fibroids. A case-control study," Obstetrics and Gynecology, vol. 72, no. 6, pp. 853-857, 1988.
[26] National Center for Health Statistics and E. McCarthy, "Inpatient utilization of short-stay hospitals, by diagnosis," Vital and Health Statistics, vol. 13, no. 69, pp. 1-82, 1982.

[27] X. Gao, J. Outley, M. Botteman, J. Spalding, J. A. Simon, and C. L. Pashos, "Economic burden of endometriosis," Fertility and Sterility, vol. 86, no. 6, pp. 1561-1572, 2006.

[28] T. Kunisue, Z. Chen, G. M. Buck Louis et al., "Urinary concentrations of benzophenone-type UV filters in U.S. women and their association with endometriosis," Environmental Science and Technology, vol. 46, no. 8, pp. 4624-4632, 2012.

[29] G. M. B. Louis, C. M. Peterson, Z. Chen et al., "Perfluorochemicals and endometriosis: the ENDO study," Epidemiology, vol. 23, no. 6, pp. 799-805, 2012.

[30] N. Rahmioglu, S. A. Missmer, G. W. Montgomery, and K. T. Zondervan, "Insights into assessing the genetics of endometriosis," Current Obstetrics and Gynecology Reports, vol. 1, no. 3, pp. 124-137, 2012.

[31] I. Tandoi, E. Somigliana, J. Riparini, S. Ronzoni, P. Vigano', and M. Candiani, "High rate of endometriosis recurrence in young women," Journal of Pediatric \& Adolescent Gynecology, vol. 24, no. 6, pp. 376-379, 2011.

[32] R. Granese, A. Perino, G. Calagna et al., "Gonadotrophinreleasing hormone analogue or dienogest plus estradiol valerate to prevent pain recurrence after laparoscopic surgery for endometriosis: a multi-center randomized trial," Acta Obstetricia et Gynecologica Scandinavica, vol. 94, no. 6, pp. 637-645, 2015.

[33] B. Kirshon, A. N. Poindexter III, and J. Fast, "Endometriosis in multiparous women," Journal of Reproductive Medicine for the Obstetrician and Gynecologist, vol. 34, no. 3, pp. 215-217, 1989.

[34] T. A. Mahmood and A. Templeton, "Prevalence and genesis of endometriosis," Human Reproduction, vol. 6, no. 4, pp. 544-549, 1991.

[35] S. T. Dodge, R. S. Pumphrey, and K. Miyazawa, "Peritoneal endometriosis in women requesting reversal of sterilization," Fertility and Sterility, vol. 45, no. 6, pp. 774-777, 1986. 


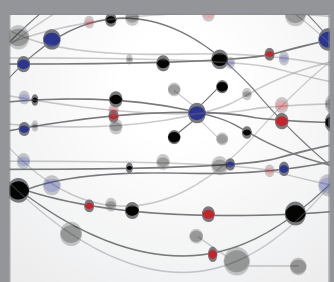

The Scientific World Journal
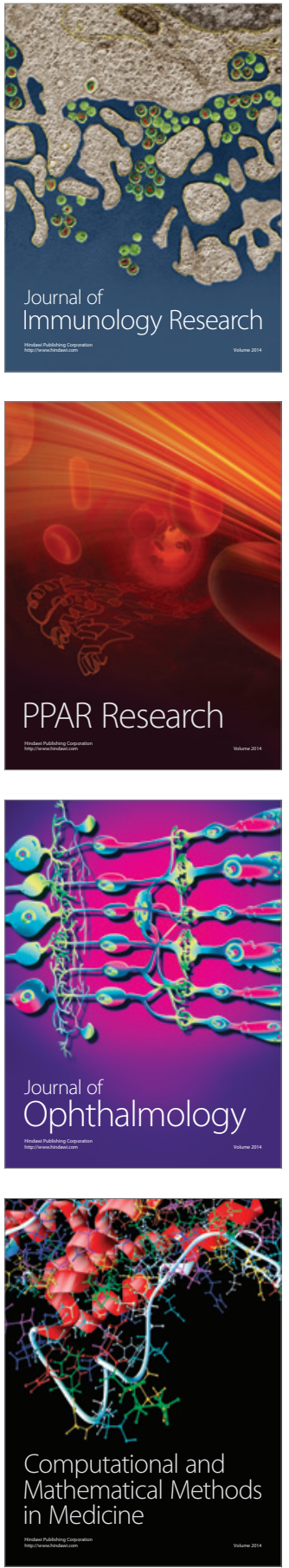

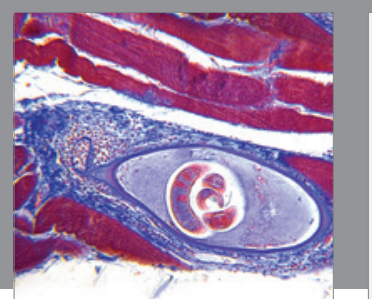

Gastroenterology Research and Practice

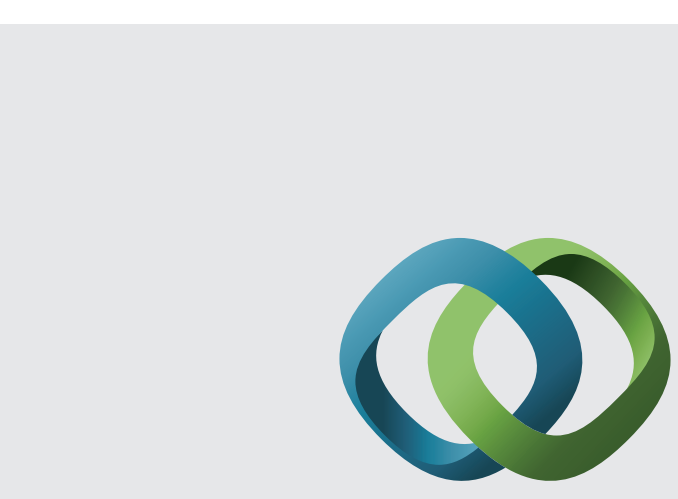

\section{Hindawi}

Submit your manuscripts at

http://www.hindawi.com
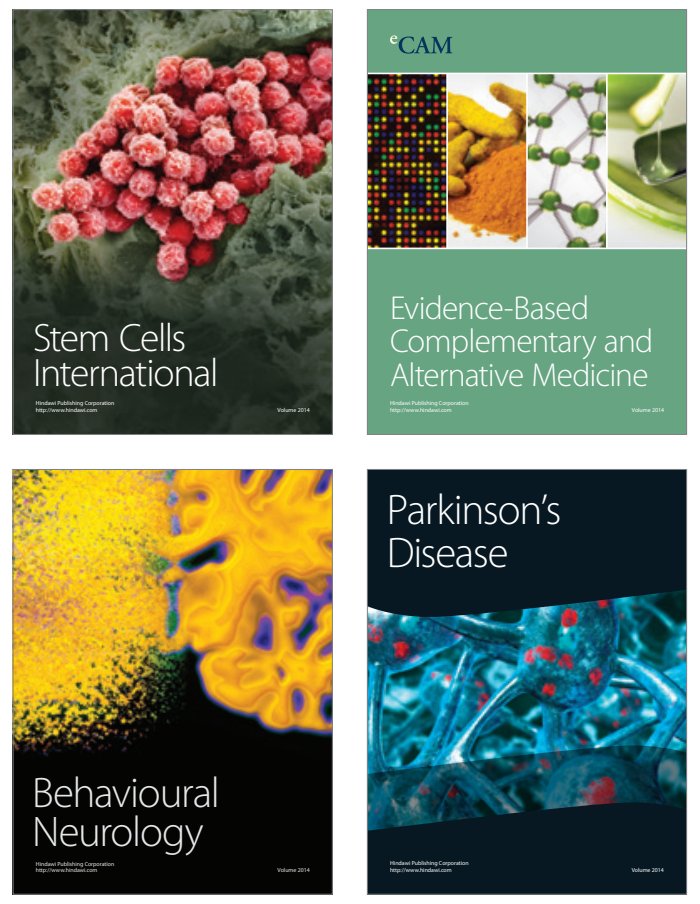
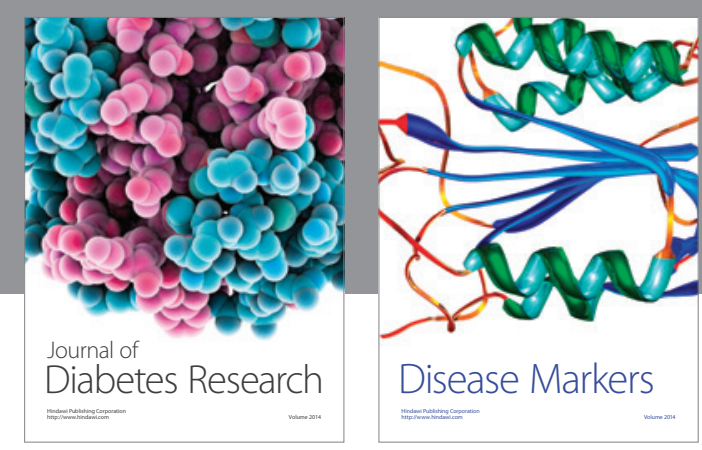

Disease Markers
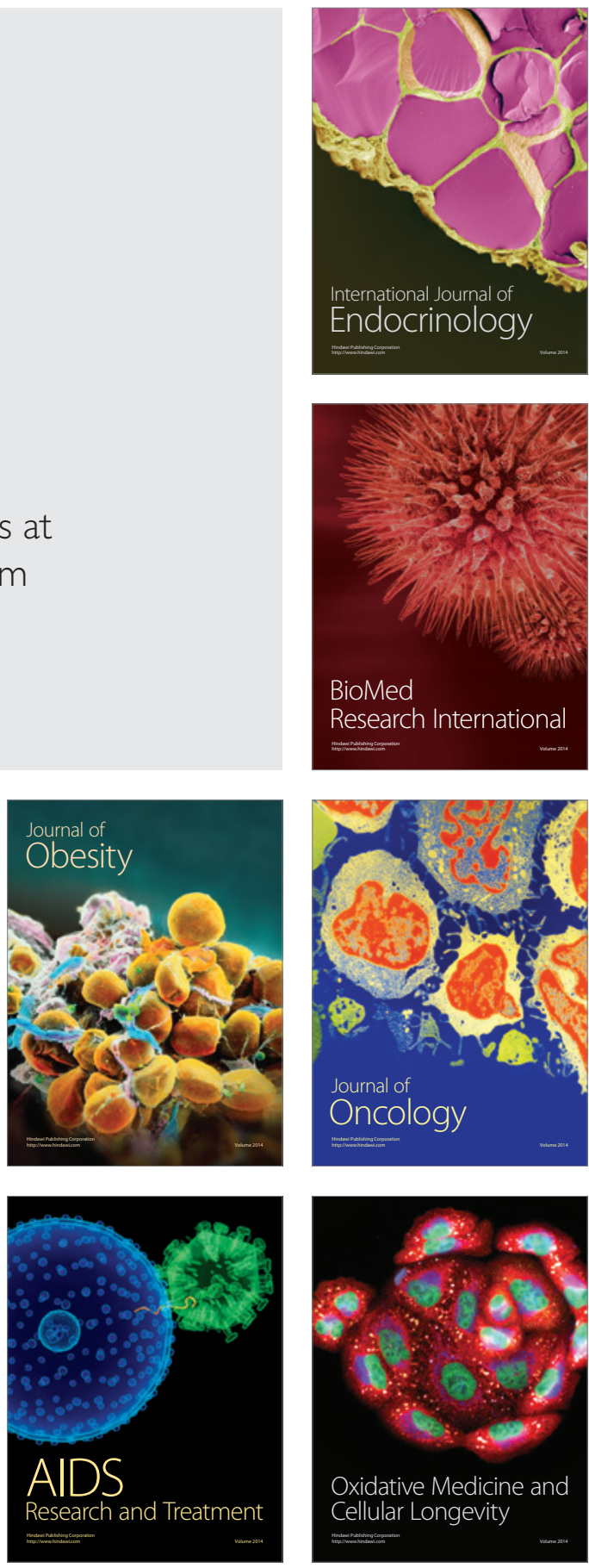